\title{
SEMI-STABLE KERNELS OF VALUATED GROUPS
}

\author{
ROBERT O. STANTON
}

\begin{abstract}
A characterization of semi-stable kernels of valuated abelian groups is given.
\end{abstract}

1. Introduction. The concept of valuated groups has recently been developed extensively by Richman and Walker [2]. (Throughout this paper, the term "group" will mean abelian group.) If $A$ is a subgroup of the group $B$, the $p$-height function of $B$ restricted to $A$ gives rise to a valuation on $A$. This relation has been quite useful in determining the structure of certain classes of groups. (For a more detailed discussion, see the introduction of [2].) Richman and Walker [1] have developed a theory of Ext in pre-abelian categories, and have applied this in [2] to valuated groups. The notions of semi-stable kernels and semi-stable cokernels are fundamental to this theory. While semi-stable cokernels are classified in a satisfactory way in [2], the question of classifying semistable kernels is left open. In this paper, a characterization of semi-stable kernels is given.

2. Valuated Groups. In this section, we summarize some definitions and results on valuated groups. Most of this discussion originated in [2]. Let $G$ be an abelian group and $p$ be a prime. The p-height function on $G$ is characterized by

$$
h_{p} x=\sup \left\{h_{p} y+1: \quad x=p y\right\}
$$

where $h_{p} x$ is either an ordinal or $\infty$. We say $\infty<\infty$ and $\alpha<\infty$ for any ordinal $\alpha$.

Definition. Let $A$ be a group and $p$ be a prime. A $p$-valuation $v_{p}$ on $A$ is a function on $A$ satisfying the following properties:

1) $v_{p} x$ is an ordinal or $\infty$

2) $v_{p}(x+y) \geqq \min \left(v_{p} x, v_{p} y\right)$

3) $v_{p} p x>v_{p} x$

4) $v_{p} n x=v_{p} x$ if $n$ is not divisible by $p$.

If $A$ is a subgroup of $B$, then the $p$-height function on $B$, restricted to $A$, is a $p$-valuation on $A$. We will restrict our study to $p$-local valuated groups,

Received by the editors on September 2, 1977, and in revised from on May 10, 1978. 
that is, valuated groups $A$ such that $A[q]=0$ and $q A=A$, whenever $q \neq p$. Thus we will drop reference to the prime $p$, and speak of the valuation $v$ on $A$, or $v_{A}$ if there is need to specify the group. If $f: A \rightarrow B$ is a one-to-one mapping such that $v f(x)=v x$ for each $a \in A$, we say $f$ is an embedding. A subgroup $A$ of $B$ is a valuated subgroup if the inclusion map is an embedding. A valuated subgroup $A$ of $B$ is called nice if each coset of $A$ contains an element of maximum value. If $\lambda$ is a function on $A$ whose values are ordinals or $\infty$, there is always a least valuation on $A$ which dominates $\lambda$. We can describe this valuation after fixing some notation. Let

$$
\begin{aligned}
& p^{\alpha} A=\{x \in A: h x \geqq \alpha\} \\
& A(\alpha)=\{x \in A: v x \geqq \alpha\} \\
& A_{\alpha}=\left\{\Sigma r_{i} x_{i}: \lambda x_{i} \geqq \alpha \text { for each } i\right\} .
\end{aligned}
$$

Lemma 1. ([2], Lemma 2.) Let $A$ be a p-local group and suppose $\lambda$ is a function on $A$ whose value is an ordinal or $\infty$. Then the smallest valuation on $A$ such that $v a \geqq \lambda a$ for each $a \in A$ is given inductively by

$$
\begin{aligned}
& A(\alpha+1)=p(A(\alpha))+A_{\alpha+1} \\
& A(\beta)=\bigcap_{\alpha<\beta} A(\alpha) \text { if } \beta \text { is a limit ordinal } \\
& v a=\sup \{\alpha: a \in A(\alpha)\} .
\end{aligned}
$$

The ( $p$-local) valuated groups form a pre-abelian category. Thus every map has a kernel and a cokernel.

TheOREM 2. ([2], Theorem 3.) If $f: A \rightarrow B$ is a map of valuated groups, then the kernel of $f$ is the group kernel with the induced valuation from $A$. The cokernel of $f$ is the group cokernel $K$ with the smallest valuation $v$ such that $v x \geqq \sup \{v b: x=\phi b\}$, where $\phi$ is the natural map of $B$ onto $K$.

A map $f: A \rightarrow B$ in the category of valuated groups is said to be a semi-stable kernel if for any pushout diagram

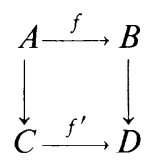

the map $f^{\prime}$ is a kernel. $f$ is a semi-stable cokernel if for any pullback diagram

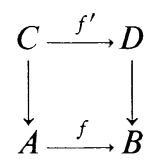


the map $f^{\prime}$ is a cokernel. A sequence $A \rightarrow^{f} B \rightarrow^{g} C$ is exact if $f=\operatorname{ker} g$ and $g=\operatorname{coker} f$. An exact sequence is stable if $f$ is a semi-stable kernel and $g$ is a semi-stable cokernel. In this case, $f$ is called a stable kernel and $g$ is a stable cokernel. The stable exact sequences constitute $\operatorname{Ext}(C, A)$.

The semi-stable kernels are those subgroups $A \subseteq B$ such that every pushout $(*)$ is an embedding. Since $C \rightarrow f^{\prime} D$ is one-to-one, we may consider $C$ as a subgroup of $D$. Thus $A \subseteq B$ is semi-stable if, whenever we have a pushout $(*), v_{C} c=v_{D} c$ for every $c \in C$.

Semi-stable cokernels are characterized by the following concept. An onto map $\phi: A \rightarrow B$ is semi-nice if whenever $b \in B$ and $\alpha<v b$, then there is $a \in A$ such that $\phi a=b$ and $v a>\alpha$.

Lemma 3. ([2], Lemma 5.) A cokernel is semi-stable if and only if it is semi-nice.

Theorem 4. ([2], Theorem 6.) The inclusion $A \subseteq B$ is a stable kernel if and only if it is a nice embedding.

The following result is a partial characterization of semi-stable kernels.

Theorem 5. ([2], Theorem 7.) If $A \subseteq B$ is a semi-stable kernel, then every coset of finite order in $B / A$ contains an element of maximum value.

Examples showing that the converse of Theorem 5 is false and that a semi-stable kernel need not be nice are given in [2]. However, the question of classifying semi-stable kernels is left open. This question will be answered by Theorem 6 .

3. Semi-Stable Kernels. In this section we characterize semi-stable kernels.

Definition. A valuated subgroup $A$ of $B$ is nearly-nice if, for each element $b \in B$ satisfying

$$
\alpha=\sup _{a \equiv A} v(b+a)>v(b+a) \text { for all } a \in A
$$

then $v\left(p^{k+1} b+a\right) \leqq \alpha+k$, for all $a \in A, k \geqq 0$.

A necessary and sufficient condition for $A$ being a valuated subgroup of $B$ that is not nearly-nice is that there is an element $b \in B$ satisfying (1) and

$$
v\left(p^{k+1} b+e\right)>\alpha+k
$$

for some $e \in A$ and $k \geqq 0$. In lieu of replacing $b$ by $p^{i} b$ and $k$ by $k-i$, where $i$ is the least integer such that $v\left(p^{i+1} b+a\right) \geqq \alpha$ for some $a \in A$, we may assume that there exists $e^{\prime} \in A$ such that

$$
v\left(p b+e^{\prime}\right) \geqq \alpha .
$$


This characterization will be useful in the following theorem.

THeorem 6. Let $A$ be a valuated subgroup of $B$. Then $A \rightarrow B$ is a semistable kernel if and only if $A$ is nearly-nice in $B$.

Proof. First assume $A$ is not nearly-nice in $B$. We will show $A \rightarrow B$ is not semi-stable. There are elements $b \in B, e \in A, e^{\prime} \in A$, and $k \geqq 0$ satisfying (1), (2) and (3). We now construct a group

$$
C=(A / A(\alpha)) \oplus[c],
$$

where $[c]$ is an infinite cyclic group. If $\phi: A \rightarrow(A / A(\alpha)) \oplus[c]$ maps $a$ to $(a+A(\alpha), 0), C$ will be valuated as follows:

$$
v_{C}\left(\phi p^{i} r e^{\prime}+p^{i+1} r c\right)=\alpha+i,
$$

whenever $i \geqq 0$ and $p \nmid r$;

$$
v_{C}(\phi a+r c)=v_{B}(a+r b)
$$

otherwise. Whenever $\phi a=0$, we must choose $a=0$ as the representative in $B$. We first show that $v_{C}$ is well defined. The hypothesis on $b$ shows that if $v_{B}(a+s b) \geqq \alpha$, then $p \mid s$. If $v_{B}(a+p s b) \geqq \alpha$, we claim that $a=s e^{\prime}+a^{\prime}$, where $a^{\prime} \in A(\alpha)$. This is true because

$$
a-s e^{\prime}=(a+p s b)-\left(s e^{\prime}+p s b\right),
$$

the difference of two elements whose value is at least $\alpha$, and so $a-s e^{\prime} \in$ $A(\alpha)$. In this case, the first formula defines the valuation of $v_{C}(\phi a+p s c)$. On the other hand, if $v_{B}\left(a_{1}+s b\right)<\alpha$ and $\phi a_{1}=\phi a_{2}$, then

$$
v_{B}\left(a_{1}+s b\right)=v_{B}\left(a_{1}-a_{2}+a_{2}+s b\right)=v_{B}\left(a_{2}+s b\right),
$$

since $v_{B}\left(a_{1}-a_{2}\right) \geqq \alpha$. So $v_{C}$ is well defined.

We now verify that $v_{C}$ is a valuation. The only condition that merits discussion is

$$
v_{C}(x+y) \geqq \min \left\{v_{C} x, v_{C} y\right\} .
$$

If $\min \left\{v_{C} x, v_{C} y\right\}<\alpha$, the condition clearly holds, so suppose $v_{C} x \geqq \alpha$, $v_{C} y \geqq \alpha$. We may write

$$
\begin{aligned}
& x=\phi p^{i} r_{1} e^{\prime}+p^{i+1} r_{1} c, p \nmid r_{1} \\
& y=\phi p^{j} r_{2} e^{\prime}+p^{j+1} r_{2} c, p \nmid r_{2}
\end{aligned}
$$

and assume $i \leqq j$. Then

$$
v_{C}(x+y)=v_{C}\left(\phi p^{i}\left(r_{1}+p^{j-i} r_{2}\right) e^{\prime}+p^{i+1}\left(r_{1}+p^{j-i} r_{2}\right) c\right) \geqq \alpha+i .
$$

Therefore $v_{C}$ is a valuation. 
Let $D$ be the valuated group completing the pushout diagram:

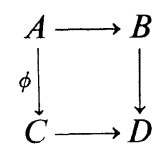

Then $D=(B \oplus C) / H$, where

$$
H=\{(a,-\phi a) \in B \oplus C: a \in A\} .
$$

The valuation on $D$ is the cokernel valuation. We wish to show $C \rightarrow D$ is not an embedding. Since $v_{C}\left(\phi p^{k} e^{\prime}+p^{k+1} c\right)=\alpha+k$, it will suffice to find some $k \geqq 0$ for which $v_{D}\left(\phi p^{k} e^{\prime}+p^{k+1} c\right)>\alpha+k$. We have

$$
\sup \left\{v_{B \oplus C}(-(b+a), \phi a+c)\right\}=\alpha,
$$

since $v_{C}(\phi a+c)=v_{B}(-(b+a))$. Therefore

$$
v_{D}\left(-p^{k+1} b+p^{k+1} c+H\right)>\alpha+k
$$

whenever $k \geqq 0$. By (2), there is $k>0$ and $e \in A$ such that

$$
v_{B}\left(p^{k+1} b+e\right)>\alpha+k .
$$

Hence $v_{D}\left(p^{k+1} b+e+H\right)>\alpha+k$. Since $e-p^{k} e^{\prime} \in A(\alpha)$, we have $\phi e=\phi p^{k} e^{\prime}$. The element

$$
-\left(p^{k+1} b+e\right)+\phi e+p^{k+1} c=-\left(p^{k+1} b+e\right)+\phi p^{k} e^{\prime}+p^{k+1} c
$$

is a representative of $-p^{k+1} b+p^{k+1} c+H$. Thus $\phi p^{k} e^{\prime}+p^{k+1} c$ is the sum of two elements of $D$, each with value greater than $\alpha+k$. Therefore

$$
v_{D}\left(\phi p^{k} e^{\prime}+p^{k+1} c\right)>\alpha+k,
$$

so $A \rightarrow B$ is not semi-stable.

Conversely, suppose $A \rightarrow B$ is not semi-stable. Then there is a pushout diagram

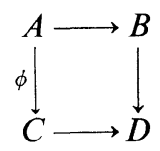

and an element $c \in C$ such that $\alpha=v_{C} c<v_{D} c . D=(B \oplus C) / H$, where $H$ is given by (4) and $D$ has the cokernel valuation. We may assume $\alpha$ is the least ordinal $\gamma$ for which there is $c^{\prime} \in C$ for which $v_{C} c^{\prime}<v_{D} c^{\prime}$. Since $v_{D}(c) \geqq \alpha+1$, we have

$$
c=p x+y
$$

where $x \in D(\alpha)$ and $y \in D_{\alpha+1}$, by Lemma 1 . There is a representative $b^{\prime}+c^{\prime}$ of $y$ such that $v_{B \oplus C}\left(b^{\prime}+c^{\prime}\right) \geqq \alpha+1$. Let $b^{\prime \prime}+c^{\prime \prime}$ be any repre- 
sentative of $x$. Considering $c$ as an element of $B \oplus C$ and splitting into components, we have

$$
\begin{aligned}
& 0=p b^{\prime \prime}+b^{\prime}+e \\
& c=p c^{\prime \prime}+c^{\prime}-\phi e,
\end{aligned}
$$

where $e$ is some element of $A$. Because $v_{B}\left(b^{\prime}\right)>\alpha$, we have

$$
v_{B}\left(p b^{\prime \prime}+e\right)>\alpha .
$$

We now wish to show $v_{B}\left(b^{\prime \prime}+a\right)<\alpha$ for all $a \in A$. First suppose $v_{B \oplus C}\left(b^{\prime \prime}+c^{\prime \prime}\right) \geqq \alpha$. Then $v_{C}\left(p c^{\prime \prime}\right) \geqq \alpha+1$ and $v_{B}\left(p b^{\prime \prime}\right) \geqq \alpha+1$. Since

$$
c=p c^{\prime \prime}+c^{\prime}+\phi\left(p b^{\prime \prime}+b^{\prime}\right)
$$

we have $v_{C}(c) \geqq \alpha+1$, a contradiction. Therefore $v_{B \oplus C}\left(b^{\prime \prime}+c^{\prime \prime}\right)<\alpha$. Now suppose $v_{B}\left(b^{\prime \prime}\right) \geqq \alpha$. Since $v_{D}\left(b^{\prime \prime}+c^{\prime \prime}+H\right) \geqq \alpha$, we have $v_{D}\left(c^{\prime \prime}\right) \geqq \alpha$. Therefore $v_{C} c^{\prime \prime} \geqq \alpha$ by the minimality of $\alpha$. This implies $v_{B \oplus C}\left(b^{\prime \prime}+c^{\prime \prime}\right)$ $\geqq \alpha$, which is again a contradiction. Hence $v_{B}\left(b^{\prime \prime}\right)<\alpha$. Since $b^{\prime \prime}+c^{\prime \prime}$ was chosen to be an arbitrary representative of $x$, we have $v_{B}\left(b^{\prime \prime}+a\right)<\alpha$ for all $a \in A$.

We now define a sequence $\left\{X_{i}\right\}$ of subsets of $B$ inductively. Let $X_{1}=$ $\left\{b^{\prime \prime}\right\}$. If $X_{n-1}$ has already been defined, let $X_{n}=\left\{b \in B: v_{B}\left(p^{j} b+h\right)>\right.$ $v_{B}(b+a)+j$, for some $h \in A, j>0$ and every $a \in A, p b+g=b_{1}+b_{2}$, where $g \in A, b_{1} \in X_{n-1}$ and satisfies

$$
v_{B}\left(p^{k} b_{1}+a_{1}\right)>\gamma+k
$$

where $a_{1}$ is an element of $A$ and

$$
\gamma=\sup _{a \in A} v_{B}(b+a),
$$

and $b_{2}$ is an element of $B$ such that $\left.v_{B} b_{2}>\gamma\right\}$. Let $X=\bigcup_{1 \leqq i<\omega} X_{i}$. If $b \in X$, and $\gamma$ is defined by (5), then there is an element $e \in A$ and $k \geqq 0$ such that $v_{B}\left(p^{k+1} b+e\right)>\gamma+k$. This clearly holds for $b^{\prime \prime}$ with $k=0$, so suppose $b \in X_{i}, i>1$. Then

$$
p^{k+1} b+p^{k} g+a_{1}=p^{k} b_{1}+a_{1}+p^{k} b_{2}
$$

is an element of the required form. Thus every element of $X$ satisfies (2). If there is an element of $X$ which also satisfies (1), the proof would be complete. So suppose that no elements of $X$ satisfy (1). Then for each $x \in X$, there is $a_{x} \in A$ such that $v_{B}\left(x+a_{x}\right)=\sup _{a \in A} v_{B}(x+a)$. Let

$$
Y=\left\{x \in X: v_{D}(x+c+H)>v_{B}\left(x+a_{x}\right) \text { for some } c \in C\right\} .
$$

Since $v_{B}\left(b^{\prime \prime}+a\right)<\alpha$ for all $a \in A$ but $v_{D}\left(b^{\prime \prime}+c^{\prime \prime}+H\right) \geqq \alpha$, we have $b^{\prime \prime} \in Y$. Hence $Y$ is a non-empty set. Let $\beta$ be the least ordinal such that $v_{B}\left(x+a_{x}\right)=\beta$ and $x \in Y$. Since $v_{D}(x+c+H) \geqq \beta+1$ for some $c \in$ $C$, we have 


$$
x+c+H \in p D(\beta)+D_{\beta+1} .
$$

Therefore we may write

$$
x+c+a_{2}-\phi a_{2}=p\left(b_{4}+c_{4}\right)+b_{3}+c_{3},
$$

where $v_{B \oplus C}\left(b_{3}+c_{3}\right) \geqq \beta+1, b_{4}+c_{4}$ is an arbitrary representative of $b_{4}+c_{4}+H$, and $a_{2} \in A$. In particular,

$$
x+a_{2}=p b_{4}+b_{3} .
$$

Thus $v_{B} p b_{4} \leqq \beta$, and $v_{B} b_{4}<\beta$. We claim $b_{4} \in X$. Since $b_{4}$ was an arbitrary representative of its coset, $v_{B}\left(b_{4}+a\right)<\beta$ for all $a \in A$. Therefore $\sup _{a \in A} v_{B}\left(b_{4}+a\right) \leqq \beta$. Since $x \in X$, there are $h \in A, j>0$ such that

$$
v_{B}\left(p^{j} x+h\right)>\beta+j,
$$

so $p b_{4}-a_{2}=x-b_{3}$ is the required representation. Moreover,

$$
v_{B}\left(p^{j+1} b_{4}-p^{j} a_{2}+h\right)>\beta+j \geqq v_{B}\left(b_{4}+a\right)+j+1
$$

for all $a \in A$. Thus $b_{4} \in X$. It is now easily seen that $b_{4} \in Y$, contradicting the minimality of $\beta$. This completes the proof of the theorem.

\section{REFERENCES}

1. F. Richman and E. Walker, Ext in Pre-Abelian Categories, Pac. J. Math., 71 (1977), 521-535.

2. F. Richman and E. Walker, Valuated Groups, J. of Algebra, 56 (1979), 145-167.

Department of Mathematics and Computer Sciences St. John's University JAMAICA, N.Y. 11439 
